\title{
Lack of Association of SP 110 Gene Polymorphisms with Pulmonary Tuberculosis in Golestan Province, Iran
}

\author{
Semira Kheiri (MSc) \\ Department of Medical Biotechnology, \\ Faculty of Advanced Medical \\ Technologies, Golestan University of \\ Medical Science, Gorgan, Iran \\ Zohreh nematollahi(MSc) \\ Metabolic Disorders Research Center, \\ Golestan University of Medical \\ Science, Gorgan, Iran \\ Naghmeh Gholipour (PhD) \\ National Institute of Genetic \\ Engineering and Biotechnology, \\ Tehran, Iran \\ Jahanbakhsh Asadi (PhD) \\ Metabolic Disorders Research Center, \\ Golestan University of Medical \\ Science, Gorgan, Iran \\ Corresponding author: Jahanbakhsh \\ Asadi \\ Email: Ja_asadi52@yahoo.com \\ Tel: +989112239011 \\ Address: Golestan University of \\ Medical Science, Gorgan, Iran \\ Received : 07 Feb 2018 \\ Revised: 02 Mar 2018 \\ Accepted: 23 Mar 2018

Semira Kheiri https://orcid.org/0000-0002-4590-
8018
Zohreh nematollahi https://orcid.org/0000-0002-
0292-6427
Naghmeh Gholipour https://orcid.org/0000-0002-
6163-4061
Jahanbakhsh Asadi https://orcid.org/0000-0001-
8930-7647

$$
8018
$$ \\ 0292-6427 \\ 6163-4061 \\ Jahanbakhsh Asadi https://orcid_org/0000-0001-
}

ABSTRACT

Background and Objectives: Mycobacterium tuberculosis is the causative agent of pulmonary tuberculosis, a main public health problem that results in 1.5 million deaths annually. A number of epidemiological studies suggested that host genetic factors could play a main role in susceptibility to tuberculosis infection.

SP110 is an interferon-induced nuclear body protein with vital roles in apoptosis, cell cycling and immunity. SP110 gene has been suggested to be a suitable candidate for limiting TB infections. Thus, we investigated the possible association between SP110 gene polymorphisms and susceptibility to tuberculosis in the Golestan Province, Iran.

Methods: We investigated the frequency of rs1135791 polymorphism of the SPI10 gene among 100 pulmonary tuberculosis patients and 100 healthy individuals who were referred to the health centers in the Golestan Province (Iran) between 2014 and 2015. Frequency of genotypes was evaluated using amplification refractory mutation systempolymerase chain reaction.

Results: The frequency distribution of TT, TC and CC genotypes among the patients was $65 \%, 31 \%$ and $4 \%$, respectively. In the control group, the frequency distribution of TT, TC and CC genotypes was $56 \%, 46 \%$ and $7 \%$, respectively. There was no significant difference in the frequency of rs 1135791 between the patients with pulmonary tuberculosis and the healthy controls $(\mathrm{P}=0.42)$.

Conclusion: Based on the results, the SP110 rs1135791 variant is not a genetic risk factor for development of pulmonary tuberculosis in Golestan Province, Iran.

Keywords: rs 1135791T, Pulmonary tuberculosis, Golestan Province.

This paper should be cited as: Kheiri S, nematollahi Z, Gholipour N, Asadi J[Lack of Association of SP110 Gene Polymorphisms with Pulmonary Tuberculosis in Golestan Province, Iran]. mljgoums. 2018; 12(3):30-33 


\section{INTRODUCTION}

Tuberculosis (TB) remains a major global health problem and is the second leading cause of death from infectious diseases (1). Two-third of affected individuals are with latent $\mathrm{TB}$ infection, but the remaining onethird can spread active TB infections, which ultimately results in 1.5 million deaths annually (2). Family-based studies have provided abundant evidence on the role of genetics in TB susceptibility, and encouraged further host genetic studies to reveal mechanism of TB susceptibility and pathogenesis (3).

Various TB studies on animal models have identified a number of suitable candidate genes for genetic association studies in humans (4). Recently, the intracellular pathogen resistance1 (IPRl) gene located on the supersusceptibility to tuberculosis 1 (sst1) locus has been shown to contribute fundamentally to innate immunity against Mycobacterium tuberculosis infection in a murine model (5). SP110 (51 $037 \mathrm{bp}$ on 2q37.1; MIM 604457) is the human homologue of IPRI and the role of its variants has been implicated in a number of diseases including TB (7). Results of the studies on SP110 gene in the individuals with TB have been contradictory. Two single nucleotide polymorphisms (SNPs) of this gene (rs3948464 and rs2114592) were found to be associated with TB in the West African population (8), whereas rs1427294 and rs1135791 have been reported to be significantly associated with TB in India (9) and China (10), respectively. However, similar studies in other populations have not found such association (7). In addition, a metaanalysis of five SNPs could not find a consistent association with TB (11).

Based on the heterogeneity of findings and the lack of enough studies on the Asian population, this study aimed to evaluate the relationship between variation in the SP110 gene and TB in Golestan Province, Iran.

\section{MATERIAL AND METHODS}

The samples were selected from individuals who were referred to the health centers in the Golestan Province (Iran) between 2014 and 2015. This case-control study was performed on 100 pulmonary TB patients (57 males, mean age: 20 years) and 100 healthy controls (50 males, mean age: 25 years). Diagnosis was made based on the standard criteria (radiological findings, positive acid-fast bacilli sputum smear and culture, and response to anti-TB drugs). All study procedures were approved by the Institutional Review Board of Golestan University of Medical Science. Written informed consent was obtained from all participants.

Genomic DNA was isolated from the whole blood samples using commercial DNA isolation kits (Pajouhan Co., Iran). DNA concentration was adjusted to $100 \mathrm{ng} / \mu \mathrm{l}$ by adding deionized water. DNA purity was evaluated by Picodrop. The DNA samples were stored at $-20{ }^{\circ} \mathrm{C}$ until analysis.

For genotyping of rs1135791, we used amplification refractory mutation systempolymerase chain (ARMS-PCR) with a forward and two reverse primers specific for the wild type and mutant variants. Two PCR reactions were performed for each sample with a normal ARMS amplification primer and the mutant ARMS primer, and the presence or absence of a PCR product indicated the presence or absence of the target allele.

ARMS-PCR primers correspond to wild-type sequence were as follows: forward consensus primer

GGGTGAATTCACAGAGGATGGGG3');

wild type reverse primer (5' TTCAGCAGCTCTCCTAGGGTCA3'). For amplification of the mutant, the wild-type reverse primer was replaced with a mutant reverse primer (5'TTCAGCAGCTCTCCTAGGGTCG3'). All primers were purchased from SinaClon BioSciences, Iran.

The PCR solution $(25 \mu \mathrm{l})$ contained $100 \mathrm{ng}$ of genomic DNA, 10× PCR buffer, 10 pmol of each primer, $10 \mathrm{nmol}$ of dNTP, $1.5 \mathrm{mmol}$ $\mathrm{Mg}^{2+}$ and $1 \mathrm{U}$ Taq polymerase.

The cycling conditions were as follows: initial denaturation at $95{ }^{\circ} \mathrm{C}$ for 5 minutes, 30 cycles of denaturation at $95{ }^{\circ} \mathrm{C}$ for 30 seconds, annealing at $62{ }^{\circ} \mathrm{C}$ for 30 seconds, extension at $72{ }^{\circ} \mathrm{C}$ for 20 seconds and final extension at 72 ${ }^{\circ} \mathrm{C}$ for 5 minutes. Later, $10 \mu \mathrm{l}$ from the PCR products was pipetted onto $2.5 \%$ agarose gel containing $0.1 \mathrm{Ag} / \mathrm{ml}$ of ethidium bromide (Gibco BRL) for gel electrophoresis and visualization of the amplified fragments.

Statistical analysis was performed using MedCalc software (Version 12.1.4.0). P-values less than 0.05 were considered statistically significant. 


\section{RESULTS}

The patients and healthy controls were separated on the basis of three possible genotypes and the presence or absence of 195 bp PCR products. In the gel electrophoresis, the samples with the TT genotype had a single band for the primer R1 (reverse oligonucleotide primer for amplification of allele T)._Samples with the heterozygous genotype (TC) had two bands for primers $1 \mathrm{R}$ and $2 \mathrm{R}$. The samples with the CC genotype had a single band for primer $2 \mathrm{R}$ (reverse oligonucleotide primer for amplification of allele C). We were able to detect both alleles
( $\mathrm{T}$ and $\mathrm{C}$ ) among the patients and control participants.

Odds ratio (OR) was calculated to assess the association between the alleles and TB infection. There was no significant difference in the frequency distribution of the alleles between the patients and the controls (Table 1). Among 100 patients, the frequency distribution of TT, TC and CC genotypes was $65 \%, 31 \%$ and $4 \%$, respectively. In the control group, the frequency distribution of TT, TC and $\mathrm{CC}$ genotypes was $57 \%, 36 \%$ and $7 \%$, respectively.

Figure 1. Detection of the rs1135791 mutation by ARMS-PCR. L: 50 bp marker, A1 and A2: normal homozygote, B1, B2, D1, and D2: heterozygous, C1 and C2: mutant homozygote

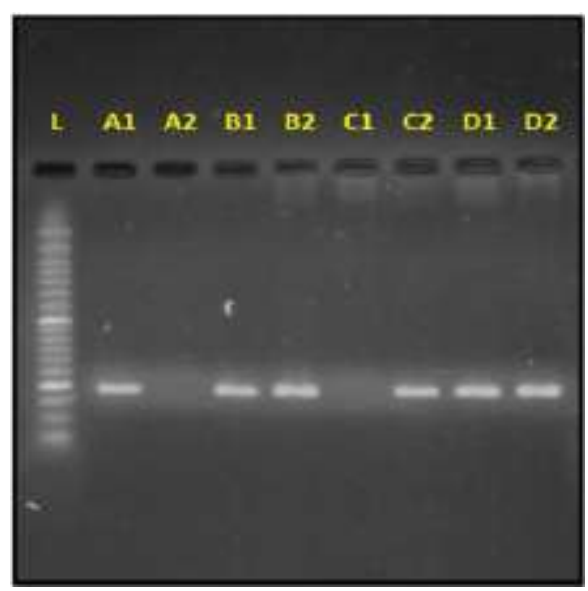

Table 1- Allele frequency distribution of the SP110SNPs

\begin{tabular}{cccccccc}
\hline Gene & Alleles & Patients $(\%)$ & Controls (\%) & OR & 95\% CI & P & X $^{2}$ \\
\hline SP110 & T & $161(80.5 \%)$ & $150(75 \%)$ & 1.0 & Reference & 0.2293 \\
SP110 & C & $39(19.5 \%)$ & $50(25 \%)$ & 1.376 & $\mathbf{0 . 8 5 7 - 2 . 2 1 1}$ & \\
\hline
\end{tabular}

\section{DISCUSSION}

TB caused by M. tuberculosis is a common and deadly infectious disease that primarily affects the lungs but can also spread to other organs, such as the kidneys and the brain. TB is more prevalent in families with a previous history of the disease, and various studies have investigated the role of host genetics in TB susceptibility. Animal studies have shown that the absence of a particular gene can lead to development of the severe form of TB. Several genetic factors have been shown to be involved in the pathogenesis of TB. Hence, studying the genetic basis of TB in different populations can provide valuable information that could be useful for treatment and prevention of the disease. One of the candidate genes for susceptibility to TB is $S P 110$, which is located on human chromosome $2 \mathrm{q} 37.1$. The gene is a member of the Sp100/Sp140 family and functions as a transcriptional activator and a nuclear hormone receptor co-activator (6). The main cytokine involved in response to viral infections is IFN (12) which itself induces SP110, indicating the role of SP110 in 
the IFN response (13). According to previous reports, controlling SP110 could be effective in controlling intracellular infections such as TB. A study on the West African population was the first to report the association of SP110 SNPs with pulmonary TB (8). However, results of our study and some previous studies in Indonesia, South and West Africa, Russia and India could not demonstrate a relationship between SP110 SNPs and susceptibility to TB (14-17). However, a study on the Chinese population found an association between rs11556887 and rs1135791 and TB susceptibility (10). As mentioned earlier, we could not find a direct relationship between SP110 SNPs and TB among Iranians living in the Golestan Province. Additional studies on the genetic basis of TB in different populations could contribute to the improvement of current

\section{REFERENCES}

1. WHO. Global tuberculosis report. 2012. World Health Organization. ISBN 9789241564502.

2. Zumla A, George A, Sharma V, Herbert N, Baroness Masham of Ilton. WHO's 2013 global report on tuberculosis: successes, threats, and opportunities. Lancet. 2013; 382(9907): 1765-7. doi: 10.1016/S0140-6736(13)62078-4.

3. Png E, Alisjahbana B, Sahiratmadja E, Marzuki S, Nelwan R, Adnan I, et al. Polymorphisms in SP110 are not associated with pulmonary tuberculosis in Indonesians. Infect Genet Evol. 2012; 12(6): 1319-23. doi: 10.1016/j.meegid.2012.04.006.

4. Qu HQ, Fisher-Hoch SP, McCormick JB. Knowledge gaining by human genetic studies on tuberculosis susceptibility. J Hum Genet. 2011; 56(3): 177-82. doi: 10.1038/jhg.2010.164.

5. Pan H, Yan BS, Rojas M, Shebzukhov YV, Zhou H, Kobzik $\mathrm{L}$, et al. Iprl gene mediates innate immunity to tuberculosis. Nature. 2005; 434(7034): 767-72.

6. Bloch DB, Nakajima A, Gulick T, Chiche JD, Orth D, de La Monte SM, et al. Sp110 localizes to the PML-Sp100 nuclear body and may function as a nuclear hormone receptor transcriptional coactivator. Mol Cell Biol2000; 20: 6138-46.

7. Fox GJ, Sy DN, Nhung NV, Yu B, Ellis MK, Van Hung N, et al. Polymorphisms of SP110 are associated with both pulmonary and extra-pulmonary tuberculosis among the Vietnamese. PloS one. 2014; 9(7): e99496.

8. Tosh K, Campbell SJ, Fielding K, Sillah J, Bah B, Gustafson $\mathrm{P}$, et al. Variants in the SP110 gene are associated with genetic susceptibility to tuberculosis in West Africa. Proc Natl Acad Sci U S A. 2006; 103(27): 10364-10368. doi: 10.1073/pnas.0603340103.

9. Abhimanyu, Jha P, Jain A, Arora K, Bose M. Genetic association study Suggests a role for SP110 variants in lymph node tuberculosis but not pulmonary tuberculosis in north Indians. Hum Immunol. 2011; 72(7): 576-80. doi: 10.1016/j.humimm.2011.03.014.. prevention and treatment methods.

\section{CONCLUSION}

Based on the results, the SP110 rs1135791 variant is not a genetic risk factor for development of pulmonary $\mathrm{TB}$ in the Golestan Province, Iran.

\section{ACKNOWLEDGEMENTS}

The authors are thankful to all participants in the study and staff of health centers in the Golestan Province. This study has been supported by a research grant from the Golestan University of Medical Sciences, Iran.

\section{CONFLICT OF INTEREST}

The authors declare that there is no conflict of interest.

10. Liang L, Zhao YL, Yue J, Liu JF, Han M, Wang H, et al. Association of SP110 gene polymorphisms with susceptibility to tuberculosis in a Chinese population. Infect Genet Evol. 2011; 11(5): 934-9. doi: 10.1016/j.meegid.2011.02.017.

11. Lei X, Zhu H, Zha L, Wang Y. SP110 gene polymorphisms and tuberculosis susceptibility: A systematic review and metaanalysis based on 10624 subjects. Infect Genet Evol. 2012; 12(7): 1473-80. doi: 10.1016/j.meegid.2012.05.011.

12. Nicewonger J, Suck G, Bloch D, Swaminathan S. EpsteinBarr virus (EBV) SM protein induces and recruits cellular Sp110b to stabilize mRNAs and enhance EBV lytic gene expression. J Virol. 2004; 78(17): 9412-22.

13. Regad T, Chelbi-Alix MK. Role and fate of PML nuclear bodies in response to interferon and viral infections. Oncogene. 2001; 20(49): 7274-86.

14. Abhimanyu, Jha P, Jain A, Arora K, Bose M. Genetic association study suggests a role for SP110 variants in lymph node tuberculosis but not pulmonary tuberculosis in north Indians. Hum Immunol. 2011; 72(7): 576-80. doi: 10.1016/j.humimm.2011.03.014.

15. Babb C, Keet EH, van Helden PD, Hoal EG. SP110 polymorphisms are not associated with pulmonary tuberculosis in a South African population. Hum Genet. 2007; 121(3-4): 5212.

16. Szeszko JS, Healy B, Stevens H, Balabanova Y, Drobniewski F, Todd JA, et al. Resequencing and association analysis of the SP110 gene in adult pulmonary tuberculosis. Hum Genet. 2007; 121(2): 155-60.

17. Thye T, Browne EN, Chinbuah MA, Gyapong J, Osei I, Owusu-Dabo E, et al. No associations of human pulmonary tuberculosis with Sp110 variants. J Med Genet. 2006; 43(7): e32. 